\title{
Soliton Solutions for Nonlinear Systems (2+1)-Dimensional Equations
}

\author{
Anwar Ja'afar Mohamad Jawad \\ Computer Engineering Technique Department, Al-Rafidain University College, Baghdad, Iraq
}

\begin{abstract}
This paper implemented two methods for solving Nonlinear systems of Partial differential equations. These are tanh method, and sine-cosine method. Methods have been successfully tested on Konopelchenko-Dubrovsky, and Dispersive Long Wave systems of equations. The calculations demonstrate the effectiveness and convenience of these two methods for solving nonlinear system of PDEs.
\end{abstract}

Keywords: Exact Solutions, Konopelchenko-Dubrovsky equations, Nonlinear system PDEs, Sine-cosine method, Tanh method,

\section{INTRODUCTION}

Nonlinear evolution equations (NLEEs) have been the subject of study in various branches of Mathematical-physical sciences such as physics, biology, and chemistry. The analytical solutions of such equations are of fundamental importance since a lot of mathematical physical models are described by NLEEs. The nonlinear wave phenomena observed in the above mentioned scientific fields, are often modeled by the bell-shaped sech solutions and the kink-shaped tanh solutions. The availability of these exact solutions, for those nonlinear equations can greatly facilitate the verification of numerical solvers on the stability analysis of the solution. The investigation of exact solutions of NLPDEs plays an important role in the study of these phenomena. In the past several decades, many effective methods for obtaining exact solutions of NLPDEs have been presented. In the literature, there is a wide variety of approaches to nonlinear problems for constructing traveling wave solutions, such as Hirota's direct method [Hirota (2004)], tanh-sech method [Malfliet (1993), Wazwaz (2006)], extended tanh method [Ma and Fuchssteiner (1996), El-Wakil et al. (2007), Fan (2000), Wazwaz (2005)], hyperbolic function method[Xia and Zhang (2001)], sine-cosine method[Wazwaz (2004), Yusufoglu and Bekir (2006)], F-expansion method [Zhang (2006)], and the transformed rational function method [Ma and Lee (2009)].

This paper outlines the implementation of efficient and reliable techniques which are Tanh method, and sine-cosine method for solving systems of coupled equations which are very important in applied sciences.

The hyperbolic tangent (tanh) method is a powerful technique to symbolically compute traveling wave solutions of evolution equations [Wazwaz (2006)]. In particular, the method is well suited for problems where dispersion, convection, and reaction diffusion phenomena play an important role.

The sine-cosine method [Mitchell(1980), Parkes (1998), Khater (2002), ] has been used to solve different types of nonlinear systems of PDEs.

\section{OUTLINE OF THE TANH METHOD}

The tanh method will be introduced as presented by [Malfliet (1992), and by Wazwaz (2006)]. The tanh method is based on a priori assumption that the traveling wave solutions can be expressed in terms of the tanh function to solve the coupled $\mathrm{KdV}$ equations.

The tanh method is developed by [Malfliet (1992)]. The method is applied to find out exact solutions of a coupled system of nonlinear differential equations with three unknowns:

$\mathrm{p}_{1}\left(\mathrm{u}, \mathrm{v}, \mathrm{u}_{\mathrm{t}}, \mathrm{v}_{\mathrm{t}}, \mathrm{u}_{\mathrm{x}}, \mathrm{v}_{\mathrm{x}}, \mathrm{u}_{\mathrm{xx}}, \mathrm{v}_{\mathrm{xy}}, \ldots \ldots \ldots\right)=0$
$\mathrm{p}_{2}\left(\mathrm{u}, \mathrm{v}, \mathrm{u}_{\mathrm{t}}, \mathrm{v}_{\mathrm{t}}, \mathrm{u}_{\mathrm{x}}, \mathrm{v}_{\mathrm{x}}, \mathrm{u}_{\mathrm{xx}}, \mathrm{v}_{\mathrm{xy}}, \ldots \ldots \ldots.\right)=0$

Where $\mathrm{p}_{1}, \mathrm{p}_{2}$ are polynomials of the variable $\mathrm{u}, \mathrm{v}$ and its derivatives. If we consider $\mathrm{u}(\mathrm{x}, \mathrm{t})=\mathrm{u}(\xi)$, $\mathrm{v}(\mathrm{x}, \mathrm{t})=\mathrm{v}(\xi)$, and let:

$\xi=\mathrm{kx}+\alpha \mathrm{y}+\omega \mathrm{t}$

so that $\mathrm{u}(\mathrm{x}, \mathrm{t})=\mathrm{U}(\xi), \mathrm{v}(\mathrm{x}, \mathrm{t})=\mathrm{V}(\xi)$ we can use the following changes:

$\frac{\partial u}{\partial x}=k \frac{d u}{d \xi}, \frac{\partial u}{\partial y}=\alpha \frac{d u}{d \xi}, \frac{\partial u}{\partial t}=\omega \frac{d u}{d \xi}, \frac{\partial^{2} u}{\partial x^{2}}=k^{2} \frac{d^{2} u}{d \xi^{2}}$

and so on, then Eq. (1) becomes an ordinary differential equation

$\mathrm{Q}_{1}\left(\mathrm{u}, \mathrm{v}, \mathrm{u}^{\prime}, \mathrm{v}^{\prime}, \mathrm{u}^{\prime \prime}, \mathrm{v}, \mathrm{v}, \ldots . . ..\right)=0$

$\mathrm{Q}_{2}\left(\mathrm{u}, \mathrm{v}^{\prime} \mathrm{u}^{\prime}, \mathrm{v}^{\prime}, \mathrm{u}^{\prime \prime}, \mathrm{v}^{\prime \prime}, \ldots \ldots . . ..\right)=0$ 
With $Q_{1}, Q_{2}$ being another polynomials form of there argument, which will be called the reduced ordinary differential equations of Eq. (4). Integrating Eq. (4) as long as all terms contain derivatives, the integration constants are considered to be zeros in view of the localized solutions. However, the nonzero constants can be used and handled as well. Now finding the traveling wave solutions to Eq. (1) is equivalent to obtaining the solution to the reduced ordinary differential equation (4). For the tanh method, we introduce the new independent variable [Malfliet (1992)]:

$\mathrm{Y}=\tanh (\xi)$

that leads to the change of variables:

$\frac{\mathrm{du}}{\mathrm{d} \xi}=\left(1-\mathrm{Y}^{2}\right) \frac{\mathrm{du}}{\mathrm{dY}}$

$\frac{\mathrm{d}^{2} \mathrm{u}}{\mathrm{d} \xi^{2}}=-2 \mathrm{Y}\left(1-\mathrm{Y}^{2}\right) \frac{\mathrm{du}}{\mathrm{dY}}+\left(1-\mathrm{Y}^{2}\right)^{2} \frac{\mathrm{d}^{2} \mathrm{u}}{\mathrm{d \textrm {Y } ^ { 2 }}}$,

$\frac{d^{2} u}{d \xi^{2}}=2\left(1-Y^{2}\right)\left(3 Y^{2}-1\right) \frac{d u}{d Y}-6 Y\left(1-Y^{2}\right)^{2} \frac{d^{2} u}{d Y^{2}}+\left(1-Y^{2}\right)^{3} \frac{d^{3} u}{d Y^{2}}$

The next crucial step is that the solution we are looking for is expressed in the form

$\mathrm{u}(\mathrm{x}, \mathrm{t})=\sum_{\mathrm{i}=0}^{\mathrm{m}} \mathrm{a}^{\mathrm{i}} \mathrm{Y}^{\mathrm{i}}, \mathrm{v}(\mathrm{x}, \mathrm{t})=\sum_{\mathrm{i}=0}^{\mathrm{m}} \mathrm{b}^{\mathrm{i}} \mathrm{Y}^{\mathrm{i}}$

where the parameters $\mathrm{m}$, and $\mathrm{n}$ can be found by balancing the highest-order linear term with the nonlinear terms in Eq. (4), and $k, \lambda_{,} a_{0}, a_{1}, \ldots, a_{m}, b_{0}, b_{1}, \ldots, b_{m}$ are to be determined. Substituting (7) into (4) will yield a set of algebraic equations for $k, \lambda, a_{0}, a_{1}, \ldots, a_{m}, b_{0}, b_{1}, \ldots, b_{m}$ because all coefficients of $Y^{i}$ have to vanish. From these relations, $\mathrm{k}, \lambda_{,} \mathrm{a}_{0}, \mathrm{a}_{1}, \ldots, \mathrm{a}_{\mathrm{m}}, \mathrm{b}_{0}, \mathrm{~b}_{1}, \ldots, \mathrm{b}_{\mathrm{m}}$ can be obtained. Having determined these parameters, knowing that $\mathrm{m}$ $, \mathrm{n}, \mathrm{s}$ are positive integers in most cases, and using (7) we obtain analytic solutions $\mathrm{u}(\mathrm{x}, \mathrm{t}), \mathrm{v}(\mathrm{x}, \mathrm{t})$ in a closed form Malfliet (1992). The tanh method seems to be powerful tool in dealing with coupled nonlinear physical models.

\section{The Sine-Cosine FunCTION Method}

Consider the nonlinear partial differential equation in the form

$\mathrm{F}\left(\mathrm{u}, \mathrm{u}_{\mathrm{t}}, \mathrm{u}_{\mathrm{x}}, \mathrm{u}_{\mathrm{y}}, \mathrm{u}_{\mathrm{tt}}, \mathrm{u}_{\mathrm{xx}}, \mathrm{u}_{\mathrm{xy}}, \mathrm{u}_{\mathrm{yy}}, \ldots \ldots \ldots\right)=0$

where $\mathrm{u}(\mathrm{x}, \mathrm{y}, \mathrm{t})$ is a traveling wave solution of nonlinear partial differential equation Eq. (8). We use the transformations,

$u(x, y, t)=f(\xi)$

Where

$\xi=\mathrm{x}+\mathrm{y}-\lambda \mathrm{t}$

This enables us to use the following changes:

$\frac{\partial}{\partial \mathrm{t}}()=.-\lambda \frac{\mathrm{d}}{\mathrm{d} \xi}(),. \frac{\partial}{\partial \mathrm{x}}()=.\frac{\mathrm{d}}{\mathrm{d} \xi}(),. \frac{\partial}{\partial \mathrm{y}}()=.\frac{\mathrm{d}}{\mathrm{d} \xi}($.

Using Eq. (10) to transfer the nonlinear partial differential equation Eq. (8) to nonlinear ordinary differential equation

$\mathrm{Q}\left(\mathrm{f}, \mathrm{f}^{\prime}, \mathrm{f}^{\mathrm{s}}, \mathrm{f}^{\mathrm{st}}\right.$ )$=0$

The ordinary differential equation (12) is then integrated as long as all terms contain derivatives, where we neglect the integration constants. The solutions of many nonlinear equations can be expressed in the form: [Ali et al (2007), Wazwaz (2004)]

$f(\xi)=\alpha \cdot \sin ^{\beta}(\mu \xi), \quad|\xi| \leq \frac{\pi}{2 \mu}$

or in the form

$f(\xi)=\alpha \cdot \cos ^{\beta}(\mu \xi), \quad|\xi| \leq \frac{\pi}{2 \mu}$

Where $\alpha, \mu$, and $\beta$ are parameters to be determined, $\mu$ and $\mathrm{c}$ are the wave number and the wave speed, respectively. Parks (1998) use

$$
\begin{aligned}
& f(\xi)=\alpha \sin ^{\beta}\left(\mu^{\xi}\right) \\
& f^{\prime}(\xi)=\alpha \beta \mu \sin ^{\beta-1}(\mu \xi) \cos (\mu \xi) \\
& f^{\prime}(\xi)=\alpha \beta(\beta-1) \mu^{2} \sin ^{\beta-2}(\mu \xi)-\alpha \beta^{2} \mu^{2} \sin ^{\beta}(\mu \xi) \\
& \text { and their derivative. Or use } \\
& f(\xi)=\alpha \cos ^{\beta}(\mu \xi) \\
& f^{\prime}(\xi)=-\alpha \beta \mu \cos ^{\xi} \beta-1(\mu \xi) \sin (\mu \xi) \\
& f^{\prime}(\xi)=\alpha \beta(\beta-1) \mu^{2} \cos ^{\xi} \beta-2(\mu \xi)-\alpha \beta^{2} \mu^{2} \cos ^{\beta}(\mu \xi) \\
& f^{\prime \prime}(\xi)=-\alpha \beta(\beta-1)(\beta-2) \mu^{3} \cos ^{\beta} \beta-3(\mu \xi) \sin (\mu \xi)+\alpha \beta^{3} \mu^{3} \cos ^{\beta-1}(\mu \xi) \sin (\mu \xi)
\end{aligned}
$$


and so on. Substitute (15) or (16) into the reduced equation (12), balance the terms of the sine functions when (15) are used, or balance the terms of the cosine functions when (16) are used, and solve the resulting system of algebraic equations by using computerized symbolic packages. We next collect all terms with the same power in $\sin ^{\mathrm{k}}\left(\mu \xi^{\xi}\right)$ or $\cos ^{\mathrm{k}}\left(\mu^{*}\right)$ and set to zero their coefficients to get a system of algebraic equations among the unknown's $\alpha, \mu$ and $\beta$, and solve the subsequent system.

\section{APPLICATIONS}

The tanh method and sine-cosine method are generalized on two systems of coupled KdV equations.

\section{KONOPELCHENKO-DUBROVSKY COUPLED SYSTEM}

$$
\begin{aligned}
& u_{t}-u_{x x x}-6 b u u_{x}+\frac{3}{2} a^{2} u^{2} u_{x}-3 v_{y}+3 a u_{x} v=0 \\
& u_{y}=v_{x}
\end{aligned}
$$

This system was studied by [N. Taghizadeh and M. Mirzazadeh (2011)] by the first integral method. By using the traveling wave transformations in $\mathrm{Eq}(2)$ and Eq.(3), The nonlinear system of partial differential equations (17) and (18) is carried to a system of ordinary differential equations

$$
\begin{aligned}
& \omega U^{\prime}-k^{3} U^{\prime \prime \prime}-6 b k U^{\prime}+\frac{3}{2} a^{2} k U^{2} U^{\prime}-3 \alpha V^{\prime}+3 a k U^{\prime} V=0 \\
& \alpha U^{\prime}=k V^{\prime}
\end{aligned}
$$

Integrating Eq.(20) once with zero constant and we postulate the tanh series, $\mathrm{Eq}(20)$ reduces to $\mathrm{V}=\frac{\alpha}{\mathrm{k}} \mathrm{U}$

Subtitute Eq.(21) in Eq.(19), then

$\omega \mathrm{U}^{\prime}-\mathrm{k}^{3} \mathrm{U}^{\prime \prime \prime}+\frac{3}{2} \mathrm{a}^{2} \mathrm{k} \mathrm{U}^{2} \mathrm{U}^{\prime}-3 \frac{\mathrm{a}^{2}}{\mathrm{k}} \mathrm{U}^{\prime}+3[\mathrm{a} \alpha-2 \mathrm{bk}] \mathrm{U} \mathrm{U}^{\prime}=0$

Integrating Eq.(22) once with zero constant, $\mathrm{Eq}(22)$ reduces to

$$
\omega \mathrm{U}-\mathrm{k}^{3} \mathrm{U}^{\prime \prime \prime}+\frac{1}{2} \mathrm{a}^{2} \mathrm{kU}^{3}-3 \frac{\mathrm{a}^{2}}{\mathrm{k}} \mathrm{U}+\frac{3}{2}[\mathrm{a} \alpha-2 \mathrm{bk}] \mathrm{U}^{2}=0
$$

\subsection{Tanh Method}

Applying tanh method with the transformaion in (5), Eq.(23) becomes:

$\omega \mathrm{U}-\mathrm{k}^{3}\left[-2 \mathrm{Y}\left(1-\mathrm{Y}^{2}\right) \frac{\mathrm{dU}}{\mathrm{dY}}+\left(1-\mathrm{Y}^{2}\right)^{2} \frac{\mathrm{d}^{2} \mathrm{U}}{\mathrm{dY^{2 }}}\right]+\frac{1}{2} \mathrm{a}^{2} \mathrm{k} \mathrm{U}^{3}-3 \frac{\mathrm{a}^{2}}{\mathrm{k}} \mathrm{U}+\frac{3}{2}[\mathrm{a} \alpha-2 \mathrm{bk}] \mathrm{U}^{2}=0$

Now to determine the parameters $\mathrm{m}$, we balance the linear term of highest-order with the highest order nonlinear terms. So, in Eq. (24) we balance $\mathrm{U}^{2}$ with $\mathrm{U}^{3}$, to obtain

$2+m=3 m \quad$, then $m=1$

The tanh method admits the use of the finite expansion for both:

$\mathrm{u}(\mathrm{x}, \mathrm{t})=\mathrm{U}(\mathrm{Y})=\mathrm{a}_{0}+\mathrm{a}_{1} \mathrm{Y} \quad, \quad \mathrm{a}_{1} \neq 0$

Substituting $\frac{\mathrm{dU}}{\mathrm{dY}}, \frac{\mathrm{d}^{2} \mathrm{U}}{\mathrm{dY}^{2}}$ in Eq. (21) to get:

$\omega\left[a_{0}+a_{1} Y\right]+2 k^{3}\left(Y-Y^{3}\right) a_{1}+\frac{1}{2} a^{2} k\left[a_{0}{ }^{3}+3 a_{0}{ }^{2} a_{1} Y+3 a_{0} a_{1}{ }^{2} Y^{2}+a_{1}{ }^{3} Y^{3}\right]-3 \frac{a^{2}}{k}\left[a_{0}+a_{1} Y\right]+$

$\frac{3}{2}[\mathrm{a} \alpha-2 \mathrm{bk}]\left[\mathrm{a}_{0}{ }^{2}+2 \mathrm{a}_{0} \mathrm{a}_{1} \mathrm{Y}+\mathrm{a}_{1}{ }^{2} \mathrm{Y}^{2}\right]=0$

then equating the coefficient of $Y^{\mathrm{i}} \mathrm{i}=0,1,2,3$ leads to the following nonlinear system of algebraic equations:

$\mathrm{Y}^{0}: \omega+\frac{1}{2} \mathrm{a}^{2} \mathrm{ka}_{0}{ }^{2}-3 \frac{\mathrm{a}^{2}}{\mathrm{k}}+\frac{3}{2}[\mathrm{a} \alpha-2 \mathrm{bk}] \mathrm{a}_{0}=0$

$\mathrm{Y}^{1}: \omega \mathrm{k}+2 \mathrm{k}^{4}+\frac{3}{2} \mathrm{a}^{2} \mathrm{k}^{2} \mathrm{a}_{0}{ }^{2}-3 \alpha^{2}+3 \mathrm{k}[\mathrm{a} \alpha-2 \mathrm{bk}] \mathrm{a}_{0}=0$

$\mathrm{Y}^{2}: \mathrm{a}^{2} \mathrm{ka} \mathrm{a}_{0}+[\mathrm{a} \alpha-2 \mathrm{bk}]=0$

$\mathrm{Y}^{3}:-2 \mathrm{k}^{2}+\frac{1}{2} \mathrm{a}^{2} \mathrm{a}_{1}{ }^{2}=0$

Solving the nonlinear systems of equations (27) we get:

$$
\alpha=\left[b-i a \frac{k}{\sqrt{2}}\right] \frac{2 k}{a}, a_{0}=\mp \sqrt{2} \frac{k}{a} i, a_{1}=\mp \frac{2 k}{a},
$$

Case 1

$$
\begin{aligned}
& \frac{\text { a }}{\mathrm{a}_{0}=\sqrt{2}} \frac{\mathrm{k}}{\mathrm{a}} \mathrm{i}, \mathrm{a}_{1}=\frac{2 \mathrm{k}}{\mathrm{a}} \\
& \mathrm{u}_{1}(\mathrm{x}, \mathrm{t})=\frac{2 \mathrm{k}}{\mathrm{a}}\left[\frac{\mathrm{i}}{\sqrt{2}}+\tanh \left(\mathrm{kx}+\left[\mathrm{b}-\mathrm{ia} \frac{\mathrm{k}}{\sqrt{2}}\right] \frac{2 \mathrm{k}}{\mathrm{a}} \mathrm{y}+\omega \mathrm{t}\right)\right] \\
& \mathrm{v}_{1}(\mathrm{x}, \mathrm{t})=\frac{4 \mathrm{k}}{\mathrm{a}^{2}}\left[\mathrm{~b}-\mathrm{ia} \frac{\mathrm{k}}{\sqrt{2}}\right]\left[\frac{\mathrm{i}}{\sqrt{2}}+\tanh \left(\mathrm{kx}+\left[\mathrm{b}-\mathrm{ia} \frac{\mathrm{k}}{\sqrt{2}}\right] \frac{2 \mathrm{k}}{\mathrm{a}} \mathrm{y}+\omega \mathrm{t}\right)\right]
\end{aligned}
$$

\section{Case 2}


$\mathrm{a}_{0}=\sqrt{2} \frac{\mathrm{k}}{\mathrm{a}} \mathrm{i}, \mathrm{a}_{1}=-\frac{2 \mathrm{k}}{\mathrm{a}}$

$\mathrm{u}_{2}(\mathrm{x}, \mathrm{t})=\frac{2 \mathrm{k}}{\mathrm{a}}\left[\frac{\mathrm{i}}{\sqrt{2}}-\tanh \left(\mathrm{kx}+\left[\mathrm{b}-\mathrm{i} \mathrm{a} \frac{\mathrm{k}}{\sqrt{2}}\right] \frac{2 \mathrm{k}}{\mathrm{a}} \mathrm{y}+\omega \mathrm{t}\right)\right]$

$\mathrm{v}_{2}(\mathrm{x}, \mathrm{t})=\frac{4 \mathrm{k}}{\mathrm{a}^{2}}\left[\mathrm{~b}-\mathrm{i} \mathrm{a} \frac{\mathrm{k}}{\sqrt{2}}\right]\left[\frac{\mathrm{i}}{\sqrt{2}}-\tanh \left(\mathrm{kx}+\left[\mathrm{b}-\mathrm{i} \mathrm{a} \frac{\mathrm{k}}{\sqrt{2}}\right] \frac{2 \mathrm{k}}{\mathrm{a}} \mathrm{y}+\omega \mathrm{t}\right)\right]$

Case 3

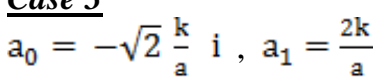

$\mathrm{u}_{3}(\mathrm{x}, \mathrm{t})=\frac{2 \mathrm{k}}{\mathrm{a}}\left[-\frac{\mathrm{i}}{\sqrt{2}}+\tanh \left(\mathrm{kx}+\left[\mathrm{b}-\mathrm{i} \mathrm{a} \frac{\mathrm{k}}{\sqrt{2}}\right] \frac{2 \mathrm{k}}{\mathrm{a}} \mathrm{y}+\omega \mathrm{t}\right)\right]$

$\mathrm{v}_{3}(\mathrm{x}, \mathrm{t})=\frac{4 \mathrm{k}}{\mathrm{a}^{2}}\left[\mathrm{~b}-\mathrm{i} \mathrm{a} \frac{\mathrm{k}}{\sqrt{2}}\right]\left[-\frac{\mathrm{i}}{\sqrt{2}}+\tanh \left(\mathrm{kx}+\left[\mathrm{b}-\mathrm{i} \mathrm{a} \frac{\mathrm{k}}{\sqrt{2}}\right] \frac{2 \mathrm{k}}{\mathrm{a}} \mathrm{y}+\omega \mathrm{t}\right)\right]$

Case 4

$\mathrm{a}_{0}=-\sqrt{2} \frac{\mathrm{k}}{\mathrm{i}}, \mathrm{a}_{1}=-\frac{2 \mathrm{k}}{\mathrm{a}}$

$\mathrm{u}_{4}(\mathrm{x}, \mathrm{t})=-\frac{2 \mathrm{k}}{\mathrm{a}}\left[\frac{\mathrm{i}}{\sqrt{2}}+\tanh \left(\mathrm{kx}+\left[\mathrm{b}-\mathrm{i} \mathrm{a} \frac{\mathrm{k}}{\sqrt{2}}\right] \frac{2 \mathrm{k}}{\mathrm{a}} \mathrm{y}+\omega \mathrm{t}\right)\right]$

$\mathrm{v}_{4}(\mathrm{x}, \mathrm{t})=-\frac{4 \mathrm{k}}{\mathrm{a}^{2}}\left[\mathrm{~b}-\mathrm{i} \mathrm{a} \frac{\mathrm{k}}{\sqrt{2}}\right]\left[\frac{\mathrm{i}}{\sqrt{2}}+\tanh \left(\mathrm{kx}+\left[\mathrm{b}-\mathrm{i} \mathrm{a} \frac{\mathrm{k}}{\sqrt{2}}\right] \frac{2 \mathrm{k}}{\mathrm{a}} \mathrm{y}+\omega \mathrm{t}\right)\right]$

\subsection{Sine-Cosine Method}

By applying Sine-cosine method to solve Eq.(23), and seeking the solution in (16) then

$2\left(k \omega-3 \alpha_{1}^{2}\right) \alpha \cos ^{\beta}(\mu \xi)-2 k^{4}\left[\alpha \beta(\beta-1) \mu^{2} \cos ^{\beta}-2(\mu \xi)-\alpha \beta^{2} \mu^{2} \cos ^{\beta}(\mu \xi)\right]+k^{2} a^{2} \alpha^{3} \cos ^{3 \beta}(\mu \xi)+$ $\left.3 \mathrm{k}\left[\mathrm{ad}_{1}-2 \mathrm{bk}\right] \alpha^{2} \cos ^{2 \beta}\left(\mu_{\zeta}\right)^{2}\right)=0$

Where $\alpha_{1} \equiv \alpha$ in (2). Equating the exponents and the coefficients of each pair of the cosine functions we find the following algebraic system:

$\beta-2=3 \beta$, then $\beta=-1$

$2\left(\mathrm{k} \omega-3 \delta^{2}\right)+2 \mathrm{k}^{4} \beta^{2} \mu^{2}=0$

$\mathrm{k}\left[\mathrm{ad}_{1}-2 \mathrm{bk}\right] \alpha^{2}=0$

$-2 \mathrm{k}^{4} \alpha \beta(\beta-1) \mu^{2}+\mathrm{k}^{2} \mathrm{a}^{2} \alpha^{3}=0$

By solving the algebraic system (36), we get,

$\mu=\frac{1}{\mathrm{ka}} \sqrt{\frac{12 \mathrm{~b}^{2} \mathrm{k}-\omega \mathrm{a}^{2}}{\mathrm{k}}}, \alpha_{1}=\frac{2 \mathrm{bl}}{\mathrm{a}}, \alpha=\frac{2}{\mathrm{a}^{2}} \sqrt{\frac{12 \mathrm{~b}^{2} \mathrm{k}-\omega \mathrm{a}^{2}}{\mathrm{k}}}$

Then by substituting Eq. (37) into Eq. (16) then, the exact soliton solution of equation (23) can be written in the form:

$\mathrm{u}(\mathrm{x}, \mathrm{y}, \mathrm{t})=\frac{2}{\mathrm{a}^{2}} \sqrt{\frac{12 \mathrm{~b}^{2} \mathrm{k}-\omega \mathrm{a}^{2}}{\mathrm{k}}} \sec \left(\frac{1}{\mathrm{ka}} \sqrt{\frac{12 \mathrm{~b}^{2} \mathrm{k}-\omega \mathrm{a}^{2}}{\mathrm{k}}}\left(\mathrm{kx}+\frac{2 \mathrm{bk}}{\mathrm{a}} \mathrm{y}+\omega \mathrm{t}\right)\right)$

and

$v(x, y, t)=\frac{4 b}{a^{3}} \sqrt{\frac{12 b^{2} k-\omega a^{2}}{k}} \sec \left(\frac{1}{k a} \sqrt{\frac{12 b^{2} k-\omega a^{2}}{k}}\left(k x+\frac{2 b k}{a} y+\omega t\right)\right)$

\section{DISPERSIVE LONG WAVE DLW EQUATIONS:}

The (2+1)- dimensional Dispersive Long Wave DLW Equations studied by [ Sheng Zhang et al (2008)], the system of equations is:

$\mathrm{u}_{\mathrm{yt}}+\mathrm{v}_{\mathrm{xx}}+\frac{1}{2}\left(\mathrm{u}^{2}\right)_{\mathrm{xy}}=0$

$\mathrm{v}_{\mathrm{t}}+\left(\mathrm{u} v+\mathrm{u}+\mathrm{u}_{\mathrm{xy}}\right)_{\mathrm{x}}=0$

Using the traveling wave transformations in Eq(2) and Eq.(3), The nonlinear system of partial differential equations (40) and (41) is carried to a system of ordinary differential equations

$\alpha \omega \mathrm{U}^{\prime \prime}+\mathrm{k}^{2} \mathrm{~V}^{\prime \prime}+\mathrm{k} \alpha\left(\mathrm{U} \mathrm{U}^{\prime}\right)^{\prime}=0$

$\omega \mathrm{V}^{\circ}+\mathrm{k}(\mathrm{UV})^{\circ}+\mathrm{kU}^{\circ}+\mathrm{k}^{2} \alpha \mathrm{U}^{\mathrm{ms}}=0$

Integrating Eq.(42) twice with zero constant, Eq(42) reduces to

$\omega \omega \mathrm{U}+\mathrm{k}^{2} \mathrm{~V}+\frac{\mathrm{k} a}{2} \mathrm{U}^{2}=0$

Integrating Eq.(43) once with zero constant, $\mathrm{Eq}(43)$ reduces to

$\omega \mathrm{V}+\mathrm{k} U \mathrm{~V}+\mathrm{kU}+\mathrm{k}^{2} \alpha \mathrm{U}^{s s}=0$

\subsection{Tanh Mehod}


we postulate the tanh series in Eq. (45)

$\omega \mathrm{V}+\mathrm{k} U \mathrm{~V}+\mathrm{kU}+\mathrm{k}^{2} \alpha\left[-2 \mathrm{Y}\left(1-\mathrm{Y}^{2}\right) \frac{\mathrm{du}}{\mathrm{dY}}+\left(1-\mathrm{Y}^{2}\right)^{2} \frac{\mathrm{d}^{2} \mathrm{u}}{\mathrm{d \textrm {Y } ^ { 2 }}}\right]=0$

to determine the parameters $\mathrm{m}$, and $\mathrm{n}$ we balance the linear term of highest-order with the highest order nonlinear terms. So, in Eq. (45) we balance U' with U V, to obtain

$\mathrm{m}+2=\mathrm{m}+\mathrm{n}$, then $\mathrm{n}=2$

while in Eq. (44) we balance $V$ with $U^{2}$, then $2 m=n$ and $m=1$.

The tanh method admits the use of the finite expansion for both:

$\mathrm{u}(\mathrm{x}, \mathrm{t})=\mathrm{U}(\mathrm{Y})=\mathrm{a}_{0}+\mathrm{a}_{1} \mathrm{Y} \quad, \quad \mathrm{a}_{1} \neq 0$

$\mathrm{v}(\mathrm{x}, \mathrm{t})=\mathrm{V}(\mathrm{Y})=\mathrm{b}_{0}+\mathrm{b}_{1} \mathrm{Y}+\mathrm{b}_{2} \mathrm{Y}^{2} \quad, \quad \mathrm{~b}_{2} \neq 0$

Substituting $\mathrm{U}^{*}, \mathrm{U}$, and $\mathrm{V}$ in Eq. (45),

$\omega\left[b_{0}+b_{1} Y+b_{2} Y^{2}\right]+k a_{0}\left[b_{0}+b_{1} Y+b_{2} Y^{2}\right]+k a_{1}\left[b_{0} Y+b_{1} Y^{2}+b_{2} Y^{3}\right]+k a_{0}+k a_{1} Y-2 k^{2} \alpha(Y-$ $\left.\mathrm{Y}^{3}\right) \mathrm{a}_{1}=0$

then equating the coefficient of $Y^{\mathrm{i}}, \mathrm{i}=0,1,2,3$ leads to the following nonlinear system of algebraic equations:

$\mathrm{Y}^{0}: \omega\left[\mathrm{b}_{0}\right]+\mathrm{ka}_{0}\left[\mathrm{~b}_{0}\right]+\mathrm{ka}_{0}=0$

$\mathrm{Y}^{1}: \omega\left[\mathrm{b}_{1}\right]+\mathrm{ka}_{0}\left[\mathrm{~b}_{1}\right]+\mathrm{ka}_{1}\left[\mathrm{~b}_{0}\right]+\mathrm{ka}_{1}-2 \mathrm{k}^{2} \alpha \mathrm{a}_{1}=0$

$\mathrm{Y}^{2}: \omega\left[\mathrm{b}_{2}\right]+\mathrm{ka}_{0}\left[\mathrm{~b}_{2}\right]+\mathrm{ka}_{1}\left[\mathrm{~b}_{1}\right]=0$

$\mathrm{Y}^{3}: \mathrm{b}_{2}+2 \mathrm{k} \alpha=0$

Substituting U, V in Eq. (44),

$\alpha \omega\left[\mathrm{a}_{0}+\mathrm{a}_{1} \mathrm{Y}\right]+\mathrm{k}^{2}\left[\mathrm{~b}_{0}+\mathrm{b}_{1} \mathrm{Y}+\mathrm{b}_{2} \mathrm{Y}^{2}\right]+\frac{\mathrm{k} a}{2}\left[\mathrm{a}_{0}+\mathrm{a}_{1} \mathrm{Y}\right]^{2}=0$

equating the coefficient of, $\mathrm{Y}^{\mathrm{i}}, \mathrm{i}=0,1,2$ leads to the following nonlinear system of algebraic equations

$\mathrm{Y}^{0}: \sigma \omega\left[\mathrm{a}_{0}\right]+\mathrm{k}^{2}\left[\mathrm{~b}_{0}\right]+\frac{\mathrm{ka}}{2}\left[\mathrm{a}_{0}^{2}\right]=0$

$\mathrm{Y}^{1}: \alpha \omega\left[\mathrm{a}_{1}\right]+\mathrm{k}^{2}\left[\mathrm{~b}_{1}\right]+\frac{\mathrm{ka}}{2}\left[2 \mathrm{a}_{0} \mathrm{a}_{1}\right]=0$

$\mathrm{Y}^{2}: \mathrm{k}^{2}\left[\mathrm{~b}_{2}\right]+\frac{\mathrm{k} a}{2}\left[\mathrm{a}_{1}{ }^{2}\right]=0$

Solving the nonlinear systems of equations (50) and (52), we get:

\section{Case 1}

$\alpha=\frac{2 k^{3}}{\left[4 k^{4}-\omega^{2}\right]}, a_{0}=-\frac{\omega}{k}, a_{1}=2 k, b_{0}=\frac{\omega^{2}}{\left[4 k^{4}-\omega^{2}\right]}, b_{1}=0, b_{2}=-\frac{4 k^{4}}{\left[4 k^{4}-\omega^{2}\right]}$

$\mathrm{u}(\mathrm{x}, \mathrm{t})=-\frac{\omega}{\mathrm{k}}+2 \mathrm{k} \tanh \left(\mathrm{kx}+\frac{2 \mathrm{k}^{3}}{\left[4 \mathrm{k}^{4}-\omega^{2}\right]} \mathrm{y}+\omega \mathrm{t}\right)$

$\mathrm{v}(\mathrm{x}, \mathrm{t})=\frac{1}{\left[4 \mathrm{k}^{4}-\omega^{2}\right]}\left[\omega^{2}-4 \mathrm{k}^{4} \tanh ^{2}\left(\mathrm{kx}+\frac{2 \mathrm{k}^{3}}{\left[4 \mathrm{k}^{4}-\omega^{2}\right]} \mathrm{y}+\omega \mathrm{t}\right)\right]$

for $\mathrm{k}=\omega=1$

$\mathrm{u}(\mathrm{x}, \mathrm{t})=-1+2 \tanh \left(\mathrm{x}+\frac{2}{3} \mathrm{y}+\mathrm{t}\right)$

$\mathrm{v}(\mathrm{x}, \mathrm{t})=\frac{1}{3}\left[1-4 \tanh ^{2}\left(\mathrm{x}+\frac{2}{3} \mathrm{y}+\mathrm{t}\right)\right]$

Figure(1), and figure(2) represent the solitary $\mathrm{u}(\mathrm{x}, \mathrm{t})$ in $(55)$, and $\mathrm{v}(\mathrm{x}, \mathrm{t})$ in (56) respectively, at $\mathrm{t}=0.5$,

$-10<x<10,-10<y<10$.

Case 2

$\alpha=\frac{2 k^{3}}{\left[4 k^{4}-\omega^{2}\right]}$

$a_{0}=-\frac{\omega}{k}, a_{1}=-2 k, b_{0}=\frac{\omega^{2}}{\left[4 k^{4}-\omega^{2}\right]}, b_{1}=0, b_{2}=-\frac{4 k^{4}}{\left[4 k^{4}-\omega^{2}\right]}$

$\mathrm{u}(\mathrm{x}, \mathrm{t})=-\left[\frac{\omega}{\mathrm{k}}+2 \mathrm{k} \tanh \left(\mathrm{kx}+\frac{2 \mathrm{k}^{3}}{\left[4 \mathrm{k}^{4}-\omega^{2}\right]} \mathrm{y}+\omega \mathrm{t}\right)\right]$

$\mathrm{v}(\mathrm{x}, \mathrm{t})=\frac{1}{\left[4 \mathrm{k}^{4}-\omega^{2}\right]}\left[\omega^{2}-4 \mathrm{k}^{4} \tanh ^{2}\left(\mathrm{kx}+\frac{2 \mathrm{k}^{3}}{\left[4 \mathrm{k}^{4}-\omega^{2}\right]} \mathrm{y}+\omega \mathrm{t}\right)\right]$

for $\mathrm{k}=\omega=1$

$\mathrm{u}(\mathrm{x}, \mathrm{t})=-\left[1+2 \tanh \left(\mathrm{x}+\frac{2}{3} \mathrm{y}+\mathrm{t}\right)\right]$ 
$\mathrm{v}(\mathrm{x}, \mathrm{t})=\frac{1}{3}\left[1-4 \tanh ^{2}\left(\mathrm{x}+\frac{2}{3} \mathrm{y}+\mathrm{t}\right)\right]$

Figure (3) represents the solitary $\mathrm{u}(\mathrm{x}, \mathrm{t})$ in $(55)$, at $\mathrm{t}=0.5,-10<x<10,-10<y<10$.

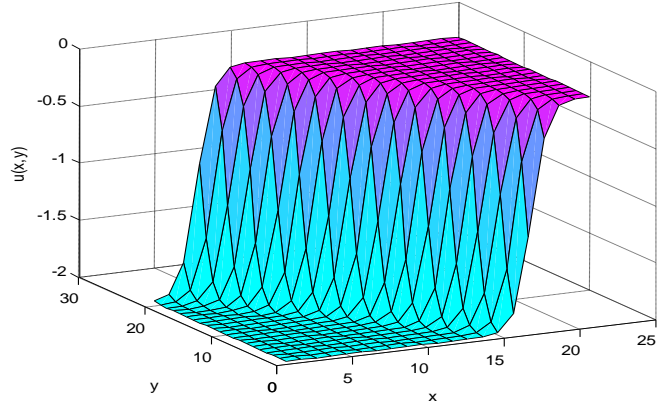

Figure (1)

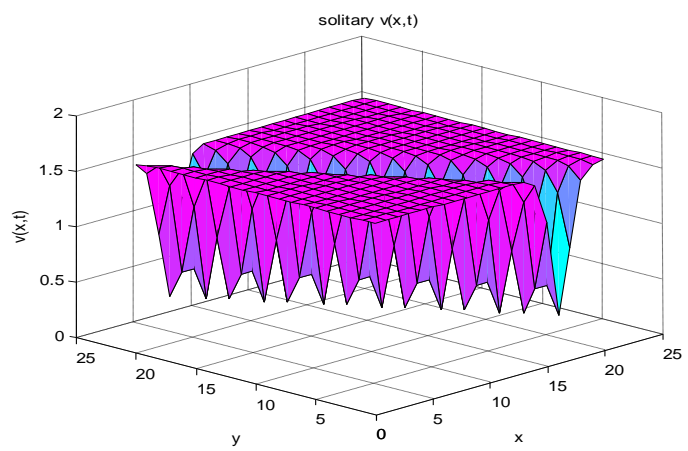

Figure (2)

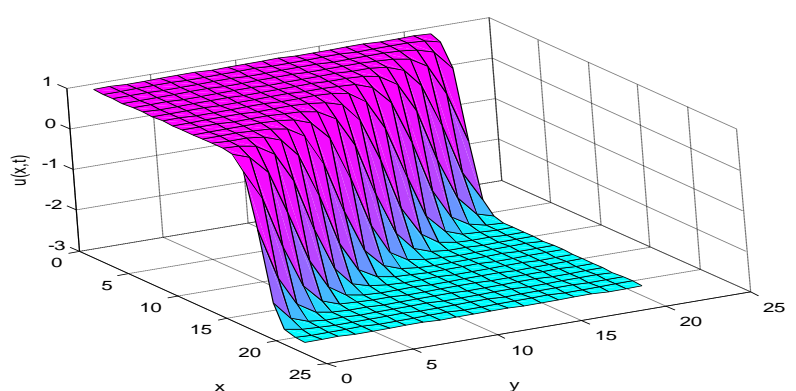

Figure (3)

\subsection{Sine-Cosine Method}

By applying Sine-cosine method to solve Eq.(42), and Eq.(43) and seeking the solution in (16) then $\mathrm{u}(\xi)=\alpha_{1} \cos ^{\beta_{1}}(\mu \xi)$

and

$\mathrm{v}(\xi)=\alpha_{2} \cos ^{\beta_{2}}(\mu \xi)$

Substituting u, u', u, v", in Eq.(42) then:

$-\alpha \omega \alpha_{1} \beta_{1}\left(\beta_{1}-1\right)\left(\beta_{1}-2\right) \mu^{3} \cos ^{\beta_{1}-3}(\mu \xi) \sin (\mu \xi)+\alpha \omega \alpha_{1} \beta_{1}^{3} \mu^{3} \cos ^{\beta_{1}-1}(\mu \xi) \sin (\mu \xi)-k^{2} \alpha_{2} \beta_{2}\left(\beta_{2}-\right.$ 1) $\left(\beta_{2}-2\right) \mu^{3} \cos ^{\beta_{2}-3}(\mu \xi) \sin (\mu \xi)+k^{2} \alpha_{2} \beta_{2}{ }^{3} \mu^{3} \cos ^{\beta_{2}-1}(\mu \xi) \sin (\mu \xi)-\mathrm{k} \alpha \alpha_{1}{ }^{2} \beta_{1}\left(\beta_{1}-1\right)\left(\beta_{1}-\right.$ 2) $\mu^{3} \cos ^{2} \beta_{1}-3(\mu \xi) \sin (\mu \xi)+\mathrm{k \alpha \alpha} \alpha_{1}{ }^{2} \beta_{1}^{3} \mu^{3} \cos ^{2 \beta_{1}-1}(\mu \xi) \sin (\mu \xi)+\mathrm{k} \alpha \alpha_{1}{ }^{2} \beta_{1}^{2} \mu^{2} \cos ^{2 \beta_{1}-2}(\mu \xi)-$ $\mathrm{k} \alpha \alpha_{1}^{2} \beta_{1}^{2} \mu^{2} \cos ^{2} \beta_{1}(\mu \xi)=0$

While Eq.(43) will be: 
$-\omega \alpha_{2} \beta_{2} \cos ^{\beta_{2}-1}(\mu \xi) \sin (\mu \xi)-\left[k \mu \alpha_{1} \alpha_{2}\left(\beta_{2}+\beta_{1}\right)\right] \sin (\mu \xi) \cos ^{\beta_{1}+\beta_{2}-1}(\mu \xi)-k^{2} \alpha_{1} \alpha \beta_{1}\left(\beta_{1}-1\right)\left(\beta_{1}-\right.$ 2) $\mu^{3} \cos ^{\beta_{1}-3}(\mu \xi) \sin (\mu \xi)+\left[\mathrm{k}^{2} \alpha \alpha_{1} \beta_{1}{ }^{3} \mu^{3}-\mathrm{k} \alpha_{1} \beta_{1}\right] \cos ^{\beta_{1}-1}(\mu \xi) \sin (\mu \xi)=0$

Equating the exponents and the coefficients of each pair of the cosine functions we find the following algebraic system:

$\beta_{1}+\beta_{2}-1=\beta_{1}-3$, then $\beta_{2}=-2$

$2 \beta_{1}=\beta_{1}-1$, then $\beta_{1}=-1$

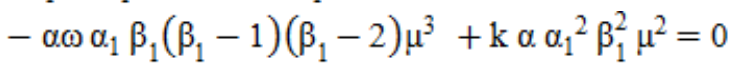

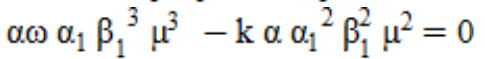

$k^{2} \alpha_{2} \beta_{2}\left(\beta_{2}-1\right)\left(\beta_{2}-2\right) \mu^{3}+\mathrm{k \alpha \alpha} \alpha_{1}^{2} \beta_{1}\left(\beta_{1}-1\right)\left(\beta_{1}-2\right) \mu^{3}=0$

$\mathrm{k}^{2} \alpha_{2} \beta_{2}{ }^{3} \mu^{3}+\mathrm{k} \alpha \alpha_{1}{ }^{2} \beta_{1}^{3} \mu^{3}=0$

While Eq.(43) will be:

$\mathrm{k} \mu \alpha_{1} \alpha_{2}\left(\beta_{2}+\beta_{1}\right)+\mathrm{k}^{2} \alpha_{1} \alpha \beta_{1}\left(\beta_{1}-1\right)\left(\beta_{1}-2\right) \mu^{3}=0$

$\mathrm{k}^{2} \alpha \cdot \alpha_{1} \beta_{1}{ }^{3} \mu^{3}-\mathrm{k} \alpha_{1} \beta_{1}=0$

By solving the algebraic systems (65), and (66), we get,

$\alpha_{1}=-2 \sqrt{2} \mathrm{k} \mu \quad, \alpha_{2}=-\frac{2}{\mu}, \omega=\frac{\sqrt{2} k^{2}}{3} \alpha=\frac{1}{k \mu^{3}}$

Then by substituting (67) into Eq. (16) then, the exact soliton solution can be written in the form:

$\mathrm{u}(\mathrm{x}, \mathrm{y}, \mathrm{t})=-2 \sqrt{2} \mathrm{k} \mu \quad \sec \left(\mu\left(\mathrm{kx}+\frac{1}{\mathrm{k \mu} \mu^{3}} \mathrm{y}+\frac{\sqrt{2} \mathrm{k}^{2}}{3} \mathrm{t}\right)\right)$

and

$\mathrm{v}(\mathrm{x}, \mathrm{y}, \mathrm{t})=-\frac{2}{\mu} \sec ^{2}\left(\mu\left(\mathrm{kx}+\frac{1}{\mathrm{k \mu} \mu^{3}} \mathrm{y}+\frac{\sqrt{2} \mathrm{k}^{2}}{3} \mathrm{t}\right)\right)$

for $\mathrm{k}=\omega=\mu=1$

$u(x, y, t)=-2 \sqrt{2} \sec \left(x+y+\frac{\sqrt{2}}{3} t\right)$

and

$v(x, y, t)=-2 \sec ^{2}\left(x+y+\frac{\sqrt{2}}{3} t\right)$

Figures (4), and (5) represent the solitary $\mathrm{u}(\mathrm{x}, \mathrm{t})$, and $\mathrm{v}(\mathrm{x}, \mathrm{t})$ in (70), and (71) respectively at $\mathrm{t}=1$, $-10<x<10,-10<y<10$.

\section{CONCLUSION}

In this paper, the tanh method and sine-cosine function method has been successfully applied to find the solution for nonlinear systems of partial differential equations. The method is used to find a new exact solution. Thus, we can say that the proposed method can be extended to solve the problems of nonlinear partial differential equations which arising in the theory of Soliton and other areas.

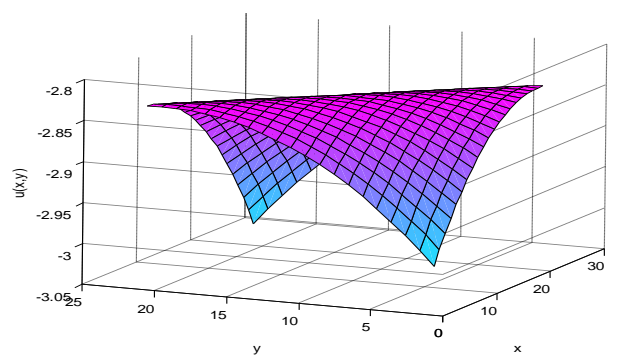

Figure (4) 


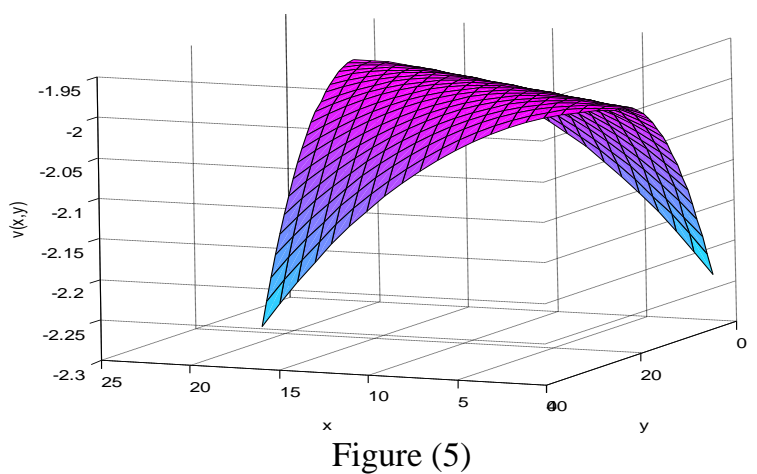

VI. ACKNOWLEDGMENT

The author gratefully acknowledges the support from the Dean of Al-Rafidain University College, Baghdad. This support is genuinely and sincerely appreciated.

\section{REFERENCES}

[1]. Ali A.H.A., A.A. Soliman , and K.R. Raslan,(2007), Soliton solution for nonlinear partial differential equations by cosine-function method, Physics Letters A 368 (2007) 299-304.

[2]. El-Wakil, S.A. and Abdou, M.A. (2007). New exact travelling wave solutions using modified extended tanh-function method, Chaos Solitons Fractals, Vol. 31, No. 4, pp. 840-852.

[3]. Fan, E. (2000). Extended tanh-function method and its applications to nonlinear equations. PhysLett A, Vol. 277, No.4, pp. 212218 .

[4]. Hirota, R. (2004). The Direct Method in Soliton Theory, Cambridge University Press. Inc, M. and Ergut, M. (2005). Periodic wave solutions for the generalized shallow water wave equation by the improved Jacobi elliptic function method, Appl. Math. E-Notes, Vol. 5, pp. 89-96.

[5]. Khater, A.H., Malfliet, W., Callebaut, D.K. and Kamel, E.S. (2002). The tanh method, a simple transformation and exact analytical solutions for nonlinear reaction-diffusion equations, Chaos Solitons Fractals, Vol. 14, No. 3, PP. 513-522.

[6]. Ma, W. X. and Lee, J. H. (2009). A transformed rational function method and exact solutions to the (3+ 1)-dimensional JimboMiwa equation, Chaos Solitons Fractals, Vol.42; pp. $1356-1363$.

[7]. Ma, W. X. and Fuchssteiner, B. (1996). Explicit and exact solutions to a Kolmogorov-Petrovskii-Piskunov equation, Int. J. NonLinear Mech. Vol. 31; pp. $329-338$

[8]. Malfliet, W. (1992). Solitary wave solutions of nonlinear wave equations, Am. J. Phys, Vol. 60,No. 7, pp. 650-654.

[9]. Mitchell A. R. and D. F. Griffiths(1980), The Finite Difference Method in Partial Differential Equations, John Wiley \& Sons.

[10]. Parkes E. J. and B. R. Duffy(1998), An automated tanh-function method for finding solitary wave solutions to nonlinear evolution equations, Comput. Phys. Commun. 98 , 288-300.

[11]. Sheng Zhang, Wei Wang, and Jing-Lin Tong, (2008), Exact Non-traveling Wave and Coefficient Function Solutions for (2+1)Dimensional Dispersive Long Wave Equations, Electronic Journal of Theoretical Physics EJTP 5, No. 19, 177-190.

[12]. Taghizadeh and M. Mirzazadeh , (2011), Exact Travelling Wave Solutions for Konopelchenko-Dubrovsky Equation by the First Integral Method, Applications and Applied Mathematics Vol. 6, Issue 11 pp. 1893-1901.

[13]. Wazwaz (2006), The tanh method for compact and non compact solutions for variants of the KdV-Burger equations, Phys. D: Nonlinear Phenomena 213 (2), 147-151.

[14]. Wazwaz, A.M. (2006). Two reliable methods for solving variants of the KdV equation with compact and noncompact structures, Chaos Solitons Fractals, Vol. 28, No. 2, pp. 454-462.

[15]. Wazwaz, A.M. (2005). The tanh-function method: Solitons and periodic solutions for the Dodd- Bullough-Mikhailov and the Tzitzeica-Dodd-Bullough equations, Chaos Solitons and Fractals, Vol. 25, No. 1, pp. 55-63.

[16]. Wazwaz, A.M. (2004). The sine-cosine method for obtaining solutions with compact and noncompact structures, Appl. Math. Comput, Vol. 159, No.2, pp. 559-576.

[17]. Xia, T.C., Li, B. and Zhang, H.Q. (2001). New explicit and exact solutions for the Nizhnik- Novikov-Vesselov equation. Appl. Math. E-Notes, Vol. 1, pp. 139-142.

[18]. Yusufoglu, E. and Bekir, A. (2006). Solitons and periodic solutions of coupled nonlinear evolution equations by using Sine-Cosine method, Internat. J. Comput. Math, Vol. 83, No. 12, pp. 915-924.

[19]. Zhang, Sheng (2006). The periodic wave solutions for the (2+1)-dimensional Konopelchenko Dubrovsky equations, Chaos Solitons Fractals, Vol. 30, pp. 1213-1220.

The Author: Dr. Anwar Ja'afar Mohamed Jawad is born in Baghdad, Iraq (1961). He is one of the academic staff in Al-Rafidain University College, Baghdad-Iraq. His designation is Assistant professor in Applied Mathematics. The academic Qualifications are PhD. in Applied Mathematics from University of Technology, Baghdad, (2000), M.Sc. in Operation Research from University of Technology, Baghdad, (1989), and B.Sc. in Mechanical Engineering from Baghdad University, (1983). He is interested in Differential equations, and Numerical analysis. He published in international journals more than 30 manuscripts in solving nonlinear partial differential equations. He was teaching Mathematics, numerical analysis for graduate and postgraduate students in Iraqi and Syrian universities. He was a supervised for many MSc. and PhD. thesis. 